\title{
Composition and temporal behavior of ambient ions in the boreal forest
}

M. Ehn ${ }^{1}$, H. Junninen ${ }^{1}$, T. Petäjä ${ }^{1}$, T. Kurtén ${ }^{1}$, V.-M. Kerminen ${ }^{1,2}, S$. Schobesberger $^{1}$, H. E. Manninen ${ }^{1}$, I. K. Ortega ${ }^{1}$, H. Vehkamäki ${ }^{1}, M$. Kulmala ${ }^{1}$, and D. R. Worsnop ${ }^{1,2,3}$

[1] \{Department of Physics, P.O.Box 64, FI-00014, University of Helsinki, Finland\}

[2] \{Finnish Meteorological Institute, Research and Development, P.O. Box 503, Fl-00101 Helsinki, Finland\}

[3] \{Aerodyne Research Inc, Billerica, M A 01821, USA\}

\section{Supplementary material}

\section{Identified ambient ions}

Below are listed all the ions that we believe to have identified during the measurements, negative ions in Table S1 and positive ions in Table S2. In some cases only the elemental composition is identified, but in most cases also the compound names are given. For the ions containing several alkyl groups, we have only listed the number of $\mathrm{C}$ atoms in the alkyl goups, as we cannot distinguish between several shorter alkyl groups and one longer, for instance dimethyl amine and ethyl amine. Grey backgrounds in Table S1 show that the ion is identified as a cluster. 


\begin{tabular}{|c|c|c|c|c|c|c|}
\hline \multirow{3}{*}{$\begin{array}{c}\text { Integer } \\
\text { mass [Da] }\end{array}$} & \multirow{3}{*}{$\begin{array}{c}\text { Elemental } \\
\text { formula }\end{array}$} & \multicolumn{3}{|c|}{ Identified ions } & \multicolumn{2}{|c|}{ Conc. $\left[\mathrm{cm}^{-3}\right]$} \\
\hline & & Chemical & Name & Exact & intege & mass \\
\hline & & formula & (adding $\mathrm{H}+$ ) & $\operatorname{mass}[\mathrm{Da}]$ & $\operatorname{Max}$ & Mean \\
\hline 45 & $\mathrm{CHO2-}$ & $\mathrm{CHO2-}$ & Formic acid & 44.9982 & 2.6 & 0.1 \\
\hline 60 & $\mathrm{CO} 3-$ & $\mathrm{CO}-$ & & 59.9853 & 2.9 & 0.1 \\
\hline 62 & O3N- & NO3- & Nitric a cid (NA) & 61.9884 & 2.9 & 0.3 \\
\hline 78 & $\mathrm{CH} 2 \mathrm{O} 4-$ & $\mathrm{H} 2 \mathrm{O} * \mathrm{CO} 3-$ & & 77.9959 & 1.9 & 0.0 \\
\hline 80 & SO3- & SO3- & & 79.9574 & 3.8 & 0.7 \\
\hline 80 & $\mathrm{H} 2 \mathrm{O} 4 \mathrm{~N}-$ & H2O*NO3- & NA + water & 79.9989 & 3.8 & 0.7 \\
\hline 87 & С3H3O3- & С3H3O3- & Pyru vic acid & 87.0088 & 2.8 & 0.5 \\
\hline 89 & $\mathrm{C} 2 \mathrm{HO} 4-$ & $\mathrm{C} 2 \mathrm{HO} 4-$ & Oxalic acid & 88.9880 & 7.3 & 1.2 \\
\hline 89 & С3H503- & C3H503- & Lactic acid & 89.0244 & 7.3 & 1.2 \\
\hline 96 & 04S- & S04- & & 95.9523 & 14.0 & 1.9 \\
\hline 97 & HO4S- & HSO4- & Sulfuric acid (SA) & 96.9601 & 242.7 & 49.1 \\
\hline 103 & С3H304- & С3H3O4- & Malonic acid (MA) & 103.0037 & 29.9 & 12.5 \\
\hline 112 & 05S- & S05- & & 111.9472 & 51.7 & 11.9 \\
\hline 113 & HO5S- & HSO5- & Peroxymonosulfuric acid & 112.9550 & 7.9 & 2.6 \\
\hline 113 & $\mathrm{C} 4 \mathrm{HO} 4-$ & $\mathrm{C} 4 \mathrm{HO} 4-$ & Squaric acid & 112.9880 & 7.9 & 2.6 \\
\hline 115 & $\mathrm{C} 4 \mathrm{H} 304-$ & $\mathrm{C} 4 \mathrm{H} 3 \mathrm{O} 4-$ & Fumaric/maleic acid (FMA) & 115.0037 & 43.2 & 4.5 \\
\hline 119 & С3H3O5- & $\mathrm{C} 3 \mathrm{H} 305-$ & Tartronic acid & 118.9986 & 2.7 & 0.6 \\
\hline 124 & $\mathrm{CH} 206 \mathrm{~N}-$ & $\mathrm{H} 2 \mathrm{CO}^{*} \mathrm{NO}^{-}$ & Carbonic acid + NA & 123.9888 & 5.0 & 1.9 \\
\hline 125 & H06N2- & HNO3*NO3- & NA dimer & 124.9840 & 34.4 & 15.1 \\
\hline 129 & C5H5O4- & C5H5O4- & Glutaconic acid & 129.0193 & 7.0 & 1.1 \\
\hline 133 & $\mathrm{C} 4 \mathrm{H} 505-$ & $\mathrm{C} 4 \mathrm{H} 505-$ & Malic acid & 133.0142 & 4.6 & 1.2 \\
\hline 142 & O6NS- & NO3*SO3- & & 141.9452 & 19.8 & 1.6 \\
\hline 147 & $\mathrm{C} 5 \mathrm{H} 705-$ & $\mathrm{C} 5 \mathrm{H} 705-$ & Hydroxypentanedioic acid & 147.0299 & 3.2 & 0.7 \\
\hline 149 & C4H506- & C4H506- & Tartaric acid & 149.0092 & 4.9 & 1.1 \\
\hline 155 & $\mathrm{C} 2 \mathrm{H} 306 \mathrm{~S}-$ & $\mathrm{C} 2 \mathrm{H} 302 \mathrm{SO} 4-$ & Glycolic acid sulfate (GAS) & 154.9656 & 6.7 & 1.4 \\
\hline 158 & 07NS- & NO3*SO4- & & 157.9401 & 6.0 & 0.8 \\
\hline 160 & H2O7NS- & HNO3*HSO4- & $\mathrm{NA}+\mathrm{SA}$ & 159.9557 & 6.6 & 1.3 \\
\hline 161 & C5H5O6- & C5H5O6- & $?$ & 161.0092 & 5.9 & 1.4 \\
\hline 166 & $\mathrm{C} 3 \mathrm{H} 407 \mathrm{~N}-$ & $\mathrm{C} 3 \mathrm{H} 404 * \mathrm{NO}_{3}-$ & $\mathrm{MA}+\mathrm{NA}$ & 165.9993 & 26.6 & 10.3 \\
\hline 175 & 031- & 103- & lodic acid & 174.8954 & 10.5 & 2.5 \\
\hline 176 & $\mathrm{C} 4 \mathrm{H} 2 \mathrm{O} 7 \mathrm{~N}-$ & C4H2O4*NO3- & $\mathrm{SqA}+\mathrm{NA}$ & 175.9837 & 5.0 & 1.0 \\
\hline 178 & $\mathrm{C} 4 \mathrm{H} 407 \mathrm{~N}-$ & C4H4O4*NO3- & $F M A+N A$ & 177.9993 & 17.3 & 2.2 \\
\hline 192 & $\mathrm{C} 5 \mathrm{H} 607 \mathrm{~N}-$ & C5H6O4*NO3- & Glutaconic acid + NA & 192.0150 & 3.6 & 1.0 \\
\hline 195 & H308S2- & $\mathrm{H} 2 \mathrm{SO} 4 * \mathrm{HSO} 4-$ & SA dimer & 194.9275 & 142.0 & 23.0 \\
\hline 201 & C3H508S- & $\mathrm{C} 3 \mathrm{H} 404 * \mathrm{HSO} 4-$ & $M A+S A$ & 200.9888 & 6.9 & 2.6 \\
\hline 207 & C6H708- & $\mathrm{C} 3 \mathrm{H} 4 \mathrm{O} 4 * \mathrm{C} 3 \mathrm{H} 3 \mathrm{O} 4-$ & Malonic acid dimer (MA2) & 207.0146 & 5.6 & 1.2 \\
\hline 211 & H3S2O9- & & & 210.9224 & 3.7 & 0.6 \\
\hline 219 & C7H708- & $\mathrm{C} 4 \mathrm{H} 4 \mathrm{O} 4 * \mathrm{C} 3 \mathrm{H} 3 \mathrm{O} 4-$ & $F M A+M A$ & 219.0146 & 3.9 & 0.6 \\
\hline 224 & C5H609N- & C5H6O6*NO3- & $?+N A$ & 224.0048 & 19.4 & 3.3 \\
\hline 231 & $\mathrm{C} 8 \mathrm{H} 708-$ & $\mathrm{C} 4 \mathrm{H} 404 * \mathrm{C} 4 \mathrm{H} 304-$ & FMA dimer & 231.0146 & 5.3 & 0.7 \\
\hline 238 & H06NI- & HNO3*IO3- & NA +lo dic acid & 237.8854 & 6.7 & 1.3 \\
\hline 253 & C2H5010S2- & $\mathrm{H} 2 \mathrm{SO}_{4} * \mathrm{C} 2 \mathrm{H} 3 \mathrm{O} 2 \mathrm{SO} 4-$ & $\mathrm{SA}+\mathrm{GAS}$ & 252.9330 & 6.7 & 1.4 \\
\hline 259 & C5H7010S- & $\mathrm{C} 3 \mathrm{H} 404 * \mathrm{C} 2 \mathrm{H} 3 \mathrm{O} 2 \mathrm{SO} 4-$ & $M A+G A S$ & 258.9765 & 5.7 & 1.1 \\
\hline 265 & С8H9010- & С5H6O6*C3H304- & $?+\mathrm{MA}$ & 265.0262 & 9.7 & 1.4 \\
\hline 293 & H5012S3- & $(\mathrm{H} 2 \mathrm{SO} 4) 2 * \mathrm{HSO} 4-$ & SA trimer & 292.8949 & 100.7 & 6.0 \\
\hline 391 & H7016S4- & (H2SO4)3*HSO4- & SA tetramer & 390.8622 & 3.8 & 1.1 \\
\hline 408 & H10016NS4- & NH3*(H2SO4)3*HSO4- & Ammonia +SA tetramer & 407.8888 & 4.8 & 1.6 \\
\hline
\end{tabular}

Table S1: List of all identified negative ion molecules (white background) and clusters (grey background). The concentrations are listed as the maximum and mean 30 min averages during April 30 - May 5. 


\begin{tabular}{|c|c|c|c|c|c|c|}
\hline \multirow{2}{*}{$\begin{array}{c}\text { Integer } \\
\text { mass [Da] }\end{array}$} & \multirow{2}{*}{$\begin{array}{c}\text { Elemental } \\
\text { formula }\end{array}$} & \multirow[b]{2}{*}{ Z } & \multirow{2}{*}{$\begin{array}{c}\text { Identified } \\
\text { compund name } \\
\text { without } \mathrm{H}+\end{array}$} & \multirow{2}{*}{$\begin{array}{c}\text { Exact } \\
\text { mass [Da] }\end{array}$} & \multicolumn{2}{|c|}{$\begin{array}{c}\text { Conc. }\left[\mathrm{cm}^{-5}\right] \\
\text { integer masses }\end{array}$} \\
\hline & & & & & Max & Mean \\
\hline 70 & C4H8N+ & $0 \mathrm{~N}$ & Pyrroline & 70.0651 & 12.0 & 4.2 \\
\hline 74 & $\mathrm{C} 4 \mathrm{H} 12 \mathrm{~N}+$ & $4 N$ & Alkyl amine (4) & 74.0964 & 3.1 & 1.0 \\
\hline 80 & $\mathrm{C} 5 \mathrm{H} 6 \mathrm{~N}+$ & $-4 N$ & Pyridine & 80.0495 & 82.1 & 36.4 \\
\hline 84 & $\mathrm{C} 5 \mathrm{H} 10 \mathrm{~N}+$ & ON & Alkyl pyrroline (1) & 84.0808 & 2.9 & 1.2 \\
\hline 88 & $\mathrm{C} 5 \mathrm{H} 14 \mathrm{~N}+$ & $4 N$ & Alkyl amine (5) & 88.1126 & 5.8 & 1.3 \\
\hline 91 & $\mathrm{C} 7 \mathrm{H} 7+$ & -7 & Tropylium ion & 91.0542 & 12.6 & 4.3 \\
\hline 92 & $\mathrm{C} 6 \mathrm{H} 6 \mathrm{~N}+$ & $-6 N$ & & 92.0495 & 3.7 & 1.6 \\
\hline 93 & $\mathrm{C} 6 \mathrm{H} 7 \mathrm{~N}+$ & $-5 N$ & & 93.0573 & 8.3 & 4.4 \\
\hline 94 & $\mathrm{C} 6 \mathrm{H} 8 \mathrm{~N}+$ & $-4 N$ & Alkyl pyridine (1) & 94.0651 & 119.1 & 57.3 \\
\hline 100 & $\mathrm{C} 5 \mathrm{H} 10 \mathrm{ON}+$ & ONO & & 100.0757 & 3.4 & 1.0 \\
\hline 102 & $\mathrm{C} 6 \mathrm{H} 16 \mathrm{~N}+$ & $4 \mathrm{~N}$ & Alkyl amine (6) & 102.1277 & 8.8 & 1.8 \\
\hline 106 & $\mathrm{C} 7 \mathrm{H} 8 \mathrm{~N}+$ & $-6 \mathrm{~N}$ & & 106.0651 & 6.0 & 3.1 \\
\hline 107 & $\mathrm{C} 7 \mathrm{H} 9 \mathrm{~N}+$ & $-5 N$ & & 107.0730 & 9.6 & 5.0 \\
\hline 108 & $\mathrm{C} 7 \mathrm{H} 10 \mathrm{~N}+$ & $-4 N$ & Alkyl pyridine (2) & 108.0808 & 74.9 & 33.5 \\
\hline 110 & $\mathrm{C} 6 \mathrm{H} 8 \mathrm{ON}+$ & $-4 N O$ & & 110.0600 & 13.2 & 6.1 \\
\hline 120 & $\mathrm{C} 8 \mathrm{H} 10 \mathrm{~N}+$ & $-6 N$ & & 120.0808 & 6.2 & 3.0 \\
\hline 121 & $\mathrm{C} 8 \mathrm{H} 11 \mathrm{~N}+$ & $-5 N$ & & 121.0886 & 3.0 & 1.4 \\
\hline 122 & $\mathrm{C} 8 \mathrm{H} 12 \mathrm{~N}+$ & $-4 N$ & Alkyl pyridine (3) & 122.0964 & 36.7 & 15.6 \\
\hline 124 & $\mathrm{C} 7 \mathrm{H} 10 \mathrm{ON}+$ & $-4 N O$ & & 124.0757 & 7.1 & 3.1 \\
\hline 128 & $\mathrm{C} 8 \mathrm{H} 18 \mathrm{~N}+$ & $2 \mathrm{~N}$ & & 128.1439 & 15.3 & 4.3 \\
\hline 130 & $\mathrm{C} 9 \mathrm{H} 8 \mathrm{~N}+$ & $-10 \mathrm{~N}$ & Quinoline & 130.0651 & 36.1 & 18.1 \\
\hline 130 & $\mathrm{C} 8 \mathrm{H} 2 \mathrm{ON}+$ & $4 \mathrm{~N}$ & Alkyl amine (8) & 130.1590 & 36.1 & 18.1 \\
\hline 136 & $\mathrm{C} 9 \mathrm{H} 14 \mathrm{~N}+$ & $-4 N$ & Alkyl pyridine (4) & 136.1121 & 58.9 & 18.5 \\
\hline 144 & $\mathrm{ClOH} 10 \mathrm{~N}+$ & $-10 \mathrm{~N}$ & Alkyl quinoline (1) & 144.0808 & 12.6 & 5.2 \\
\hline 150 & $\mathrm{ClOH} 16 \mathrm{~N}+$ & $-4 N$ & Alkyl pyridine (5) & 150.1277 & 12.8 & 5.5 \\
\hline 152 & $\mathrm{C} 9 \mathrm{H} 140 \mathrm{~N}+$ & $-4 N O$ & & 152.1070 & 3.8 & 1.7 \\
\hline 158 & $\mathrm{C} 11 \mathrm{H} 12 \mathrm{~N}+$ & $-10 N$ & Alkyl quilnoline (2) & 158.0964 & 7.1 & 3.5 \\
\hline 170 & $\mathrm{C} 12 \mathrm{H} 12 \mathrm{~N}+$ & $-12 N$ & & 170.0964 & 4.1 & 1.7 \\
\hline
\end{tabular}

Table S2: List of all identified positive ion molecules. Compound groups are mainly colored according to the Kendrick diagram (Figure 4). The concentrations are listed as the maximum and mean 30 min averages during May 5 - 8. Numbers in parenthesis after the compound names signify the number of $\mathrm{C}$ atoms in the added alkyl groups.

\section{List of all detected ions}

The software package tofTools was used to analyze the data from Hyytiälä. Below are two tables summarizing the $\mathrm{m} / \mathrm{Q}$ and mean concentrations of all the output peaks for both polarities (negative ions in S3 and positive ions in S4). Some of the peaks have been identified above, but some remain unidentified. 


\begin{tabular}{|c|c|c|c|c|c|c|c|}
\hline $\begin{array}{l}\text { Measured } \\
\text { mass [Da] }\end{array}$ & $\begin{array}{l}\text { Normalized } \\
\text { mean conc. }\end{array}$ & $\begin{array}{l}\text { Measured } \\
\text { mass [Da] }\end{array}$ & $\begin{array}{l}\text { Normalized } \\
\text { mean conc. }\end{array}$ & $\begin{array}{l}\text { Measured } \\
\text { mass [Da] }\end{array}$ & $\begin{array}{l}\text { Normalized } \\
\text { mean conc. }\end{array}$ & $\begin{array}{l}\text { Measured } \\
\text { mass [Da] }\end{array}$ & $\begin{array}{l}\text { Normalized } \\
\text { mean conc. }\end{array}$ \\
\hline 79.957 & 0.01 & 192.018 & 0.02 & 284.044 & 0.01 & 342.067 & 0.14 \\
\hline 88.990 & 0.02 & 194.928 & 0.31 & 285.024 & 0.01 & 343.061 & 0.04 \\
\hline 95.950 & 0.05 & 196.927 & 0.03 & 292.894 & 0.04 & 344.057 & 0.02 \\
\hline 96.958 & 0.95 & 197.009 & 0.01 & 294.078 & 0.02 & 345.036 & 0.01 \\
\hline 97.956 & 0.01 & 201.007 & 0.03 & 295.064 & 0.02 & 355.071 & 0.05 \\
\hline 98.954 & 0.05 & 207.019 & 0.02 & 296.069 & 0.02 & 356.060 & 0.05 \\
\hline 103.003 & 0.31 & 212.997 & 0.01 & 297.049 & 0.02 & 357.066 & 0.06 \\
\hline 111.945 & 0.25 & 217.015 & 0.02 & 298.048 & 0.02 & 358.068 & 0.08 \\
\hline 112.986 & 0.04 & 220.040 & 0.01 & 299.038 & 0.02 & 359.056 & 0.03 \\
\hline 113.943 & 0.01 & 224.013 & 0.04 & 300.035 & 0.02 & 360.058 & 0.01 \\
\hline 115.007 & 0.13 & 231.035 & 0.01 & 307.084 & 0.01 & 361.027 & 0.01 \\
\hline 118.994 & 0.01 & 234.047 & 0.02 & 308.068 & 0.16 & 370.071 & 0.02 \\
\hline 123.988 & 0.04 & 236.040 & 0.01 & 309.064 & 0.04 & 371.071 & 0.02 \\
\hline 124.985 & 0.36 & 238.041 & 0.01 & 310.072 & 0.05 & 372.051 & 0.11 \\
\hline 129.027 & 0.02 & 239.028 & 0.01 & 311.066 & 0.03 & 373.056 & 0.05 \\
\hline 131.002 & 0.01 & 240.006 & 0.03 & 312.063 & 0.02 & 374.060 & 0.03 \\
\hline 133.012 & 0.02 & 241.004 & 0.01 & 313.050 & 0.01 & 375.035 & 0.02 \\
\hline 141.946 & 0.03 & 248.057 & 0.01 & 314.044 & 0.02 & 377.046 & 0.01 \\
\hline 149.012 & 0.02 & 250.055 & 0.02 & 316.015 & 0.01 & 386.070 & 0.01 \\
\hline 154.016 & 0.02 & 252.038 & 0.01 & 323.066 & 0.02 & 387.060 & 0.06 \\
\hline 154.962 & 0.02 & 255.019 & 0.02 & 324.069 & 0.02 & 388.060 & 0.03 \\
\hline 157.955 & 0.01 & 264.058 & 0.01 & 325.068 & 0.04 & 389.049 & 0.01 \\
\hline 157.941 & 0.02 & 265.033 & 0.01 & 326.063 & 0.07 & 390.053 & 0.01 \\
\hline 159.958 & 0.02 & 266.044 & 0.01 & 327.058 & 0.02 & 402.073 & 0.01 \\
\hline 161.024 & 0.02 & 267.037 & 0.01 & 328.056 & 0.03 & 403.059 & 0.02 \\
\hline 166.002 & 0.19 & 268.035 & 0.01 & 329.038 & 0.02 & 404.051 & 0.01 \\
\hline 174.892 & 0.04 & 269.034 & 0.02 & 330.046 & 0.01 & 407.038 & 0.01 \\
\hline 175.991 & 0.01 & 281.034 & 0.02 & 339.070 & 0.04 & 408.094 & 0.01 \\
\hline 178.006 & 0.05 & 282.038 & 0.05 & 340.060 & 0.20 & 478.153 & 0.01 \\
\hline 182.013 & 0.02 & 283.042 & 0.02 & 341.063 & 0.05 & 494.157 & 0.03 \\
\hline 182.998 & 0.01 & & & & & & \\
\hline
\end{tabular}

Table S3. Anion $\mathrm{m} / \mathrm{Q}$ and mean concentration as given by tofTools during 1-5 M ay, 2009. The concentrations are normalized so that the maximum peak is 1.00 . Only peaks above 0.005 are listed. 


\begin{tabular}{|c|c|c|c|c|c|c|c|}
\hline $\begin{array}{l}\text { Measured } \\
\text { mass [Da] }\end{array}$ & $\begin{array}{l}\text { Normalized } \\
\text { mean conc. }\end{array}$ & $\begin{array}{l}\text { Measured } \\
\text { mass [Da] }\end{array}$ & $\begin{array}{l}\text { Normalized } \\
\text { mean conc. }\end{array}$ & $\begin{array}{l}\text { Measured } \\
\text { mass [Da] }\end{array}$ & $\begin{array}{l}\text { Normalized } \\
\text { mean conc. }\end{array}$ & $\begin{array}{l}\text { Measured } \\
\text { mass [Da] }\end{array}$ & $\begin{array}{l}\text { Normalized } \\
\text { mean conc. }\end{array}$ \\
\hline 70.065 & 0.07 & 139.091 & 0.0 & 213.137 & 0.01 & 286.197 & 0.03 \\
\hline 71.013 & 0.03 & 141.078 & 0.0 & 214.252 & 0.06 & 288.197 & 0.03 \\
\hline 74.093 & 0.01 & 143.081 & 0.0 & 215.120 & 0.01 & 290.184 & 0.02 \\
\hline 78.034 & 0.02 & 144.081 & 0.1 & 215.251 & 0.01 & 292.182 & 0.01 \\
\hline 80.049 & 0.71 & 144.944 & 0.0 & 216.140 & 0.01 & 294.186 & 0.01 \\
\hline 81.053 & 0.05 & 145.086 & 0.0 & 217.143 & 0.01 & 295.195 & 0.01 \\
\hline 82.987 & 0.03 & 148.100 & 0.0 & 218.142 & 0.01 & 296.209 & 0.01 \\
\hline 83.055 & 0.02 & 150.122 & 0.1 & 219.158 & 0.01 & 297.186 & 0.02 \\
\hline 84.080 & 0.02 & 151.113 & 0.0 & 221.154 & 0.02 & 298.209 & 0.02 \\
\hline 87.043 & 0.03 & 152.103 & 0.0 & 222.157 & 0.01 & 300.208 & 0.02 \\
\hline 88.109 & 0.02 & 153.098 & 0.0 & 223.159 & 0.02 & 302.196 & 0.03 \\
\hline 91.054 & 0.08 & 155.101 & 0.0 & 224.179 & 0.04 & 304.199 & 0.02 \\
\hline 92.050 & 0.02 & 156.087 & 0.0 & 225.156 & 0.02 & 306.194 & 0.01 \\
\hline 93.058 & 0.07 & 157.090 & 0.0 & 227.153 & 0.02 & 310.216 & 0.01 \\
\hline 94.065 & 1.00 & 158.096 & 0.0 & 230.158 & 0.01 & 312.218 & 0.04 \\
\hline 95.066 & 0.09 & 159.101 & 0.0 & 232.143 & 0.01 & 313.213 & 0.01 \\
\hline 96.045 & 0.03 & 163.120 & 0.0 & 234.139 & 0.01 & 314.218 & 0.03 \\
\hline 98.986 & 0.02 & 164.136 & 0.03 & 235.164 & 0.02 & 316.210 & 0.02 \\
\hline 100.079 & 0.01 & 165.115 & 0.02 & 237.174 & 0.03 & 318.200 & 0.02 \\
\hline 102.127 & 0.02 & 166.112 & 0.01 & 238.173 & 0.01 & 320.203 & 0.02 \\
\hline 105.069 & 0.01 & 167.105 & 0.02 & 239.167 & 0.04 & 322.211 & 0.01 \\
\hline 106.065 & 0.05 & 169.115 & 0.03 & 240.176 & 0.01 & 326.227 & 0.02 \\
\hline 106.947 & 0.02 & 170.097 & 0.02 & 241.159 & 0.01 & 328.225 & 0.03 \\
\hline 107.074 & 0.06 & 171.113 & 0.02 & 242.161 & 0.01 & 330.215 & 0.03 \\
\hline 108.081 & 0.52 & 172.112 & 0.02 & 244.174 & 0.02 & 332.220 & 0.02 \\
\hline 108.925 & 0.05 & 178.143 & 0.01 & 246.165 & 0.03 & 334.207 & 0.02 \\
\hline 109.082 & 0.06 & 179.065 & 0.01 & 248.153 & 0.01 & 336.209 & 0.01 \\
\hline 110.060 & 0.09 & 179.133 & 0.01 & 249.179 & 0.01 & 338.212 & 0.01 \\
\hline 111.062 & 0.01 & 180.084 & 0.01 & 250.152 & 0.01 & 340.231 & 0.02 \\
\hline 114.097 & 0.01 & 181.116 & 0.02 & 251.171 & 0.02 & 342.236 & 0.02 \\
\hline 115.058 & 0.02 & 182.134 & 0.01 & 252.190 & 0.01 & 344.228 & 0.02 \\
\hline 117.063 & 0.01 & 183.090 & 0.04 & 253.179 & 0.03 & 346.224 & 0.04 \\
\hline 119.080 & 0.01 & 184.114 & 0.01 & 254.193 & 0.02 & 347.213 & 0.01 \\
\hline 120.077 & 0.04 & 185.114 & 0.02 & 255.173 & 0.01 & 348.218 & 0.01 \\
\hline 121.087 & 0.02 & 186.132 & 0.01 & 256.186 & 0.03 & 350.218 & 0.01 \\
\hline 122.096 & 0.23 & 187.107 & 0.01 & 258.174 & 0.02 & 356.245 & 0.02 \\
\hline 123.098 & 0.04 & 192.146 & 0.01 & 260.168 & 0.02 & 358.235 & 0.02 \\
\hline 124.080 & 0.04 & 193.140 & 0.02 & 262.164 & 0.02 & 360.230 & 0.02 \\
\hline 125.078 & 0.01 & 195.129 & 0.01 & 266.189 & 0.01 & 362.227 & 0.02 \\
\hline 126.938 & 0.09 & 197.127 & 0.01 & 268.197 & 0.02 & 364.226 & 0.01 \\
\hline 127.074 & 0.01 & 199.131 & 0.02 & 269.180 & 0.02 & 370.246 & 0.01 \\
\hline 128.141 & 0.1 & 200.131 & 0.01 & 270.196 & 0.03 & 372.243 & 0.01 \\
\hline 128.063 & 0.0 & 201.123 & 0.01 & 271.197 & 0.01 & 374.248 & 0.01 \\
\hline 130.066 & 0.2 & 202.137 & 0.01 & 272.190 & 0.02 & 376.239 & 0.01 \\
\hline 131.072 & 0.0 & 203.135 & 0.01 & 274.177 & 0.02 & 378.226 & 0.01 \\
\hline 134.088 & 0.0 & 205.140 & 0.02 & 276.173 & 0.02 & 386.253 & 0.01 \\
\hline 135.100 & 0.0 & 207.157 & 0.05 & 277.159 & 0.01 & 388.262 & 0.01 \\
\hline 136.112 & 0.3 & 208.159 & 0.01 & 278.172 & 0.01 & 390.247 & 0.01 \\
\hline 136.994 & 0.1 & 209.146 & 0.02 & 279.167 & 0.01 & 398.256 & 0.01 \\
\hline 137.111 & 0.0 & 210.151 & 0.02 & 280.186 & 0.01 & 402.258 & 0.01 \\
\hline 138.094 & 0.0 & 211.145 & 0.02 & 284.198 & 0.01 & 404.248 & 0.01 \\
\hline
\end{tabular}

Table S4. Cation $\mathrm{m} / \mathrm{Q}$ and mean concentration as given by tofTools during 6-7 May, 2009. The concentrations are normalized so that the maximum peak is 1.00 . Only peaks above 0.005 are listed. 


\section{Additional figures}
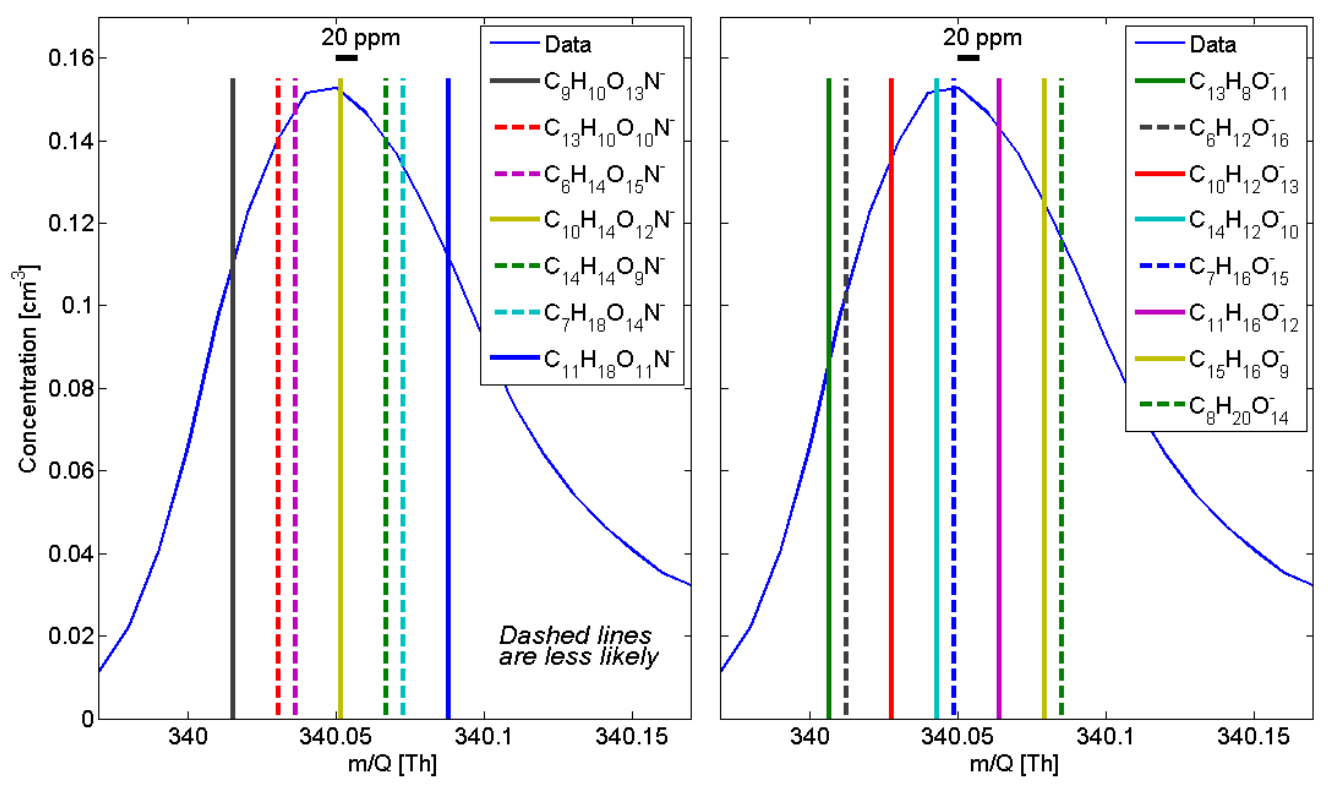

Figure S1. Average mass spectrum around m/Q 340 during $2 \mathrm{M}$ ay, 2009. In the left panel all $\mathrm{C}_{c} \mathrm{H}_{h} \mathrm{O}_{0} \mathrm{~N}^{-}$ compounds with masses between 340.000 and 340.100 are plotted, and in the right panel all $\mathrm{C}_{\mathrm{C}} \mathrm{H}_{\mathrm{h}} \mathrm{O}_{0}$ in the same $\mathrm{m} / \mathrm{Q}$ range. This figure illustrates the amount of compounds that are possible at this $\mathrm{m} / \mathrm{Q}$. According to the nitrogen rule an even $\mathrm{m} / \mathrm{Q}$ peak must include an odd number of $\mathrm{N}$, thus the most likely compound at this mass was proposed to be $\mathrm{C}_{10} \mathrm{H}_{14} \mathrm{O}_{12} \mathrm{~N}$. The best estimate for the exact mass of this peak was determined from many different time periods, including periods when small amount of iodine was mixed with the sample, to get reference peaks at higher masses (mainly $I_{3}$ at 380.713 Th). For these plots, 340.058 was used as one of the m/Q calibration peaks. However, considering the $20 \mathrm{ppm}$ accuracy of the instrument, the $\mathrm{m} / \mathrm{Q}$ calibration would have to be $100 \mathrm{pm}$ off to fit either of the two other reasonable options in the left panel. If, for some reason, the nitrogen rule does not apply for this ion, the amount of possibilities increases. And finally, if the ion contains other compounds than $\mathrm{C}, \mathrm{H}, \mathrm{O}$ and $\mathrm{N}$, the possibilities also increase. 


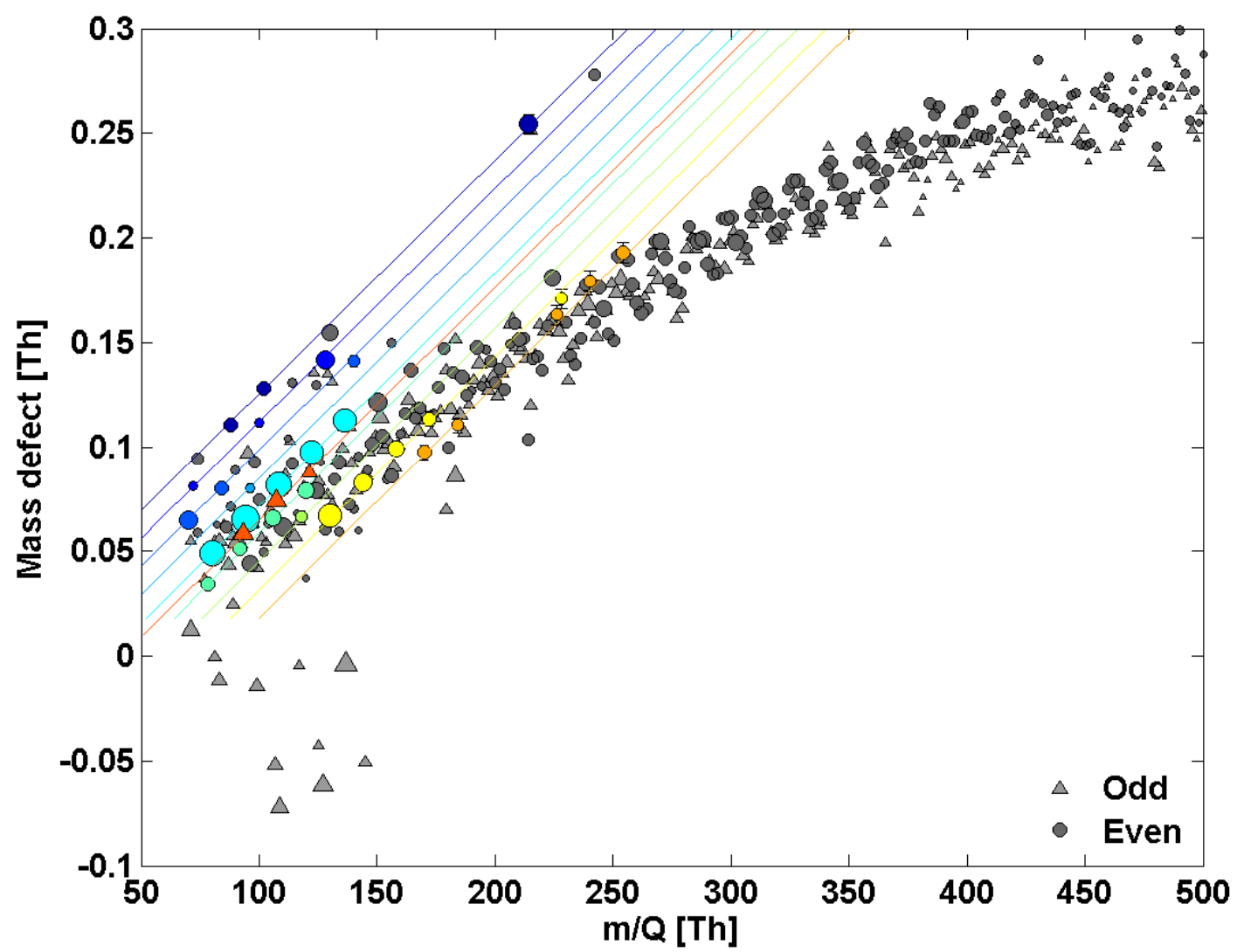

Figure S2. M ass defects as a function of m/ Q for the positive ion in Hyytiälä during 6-7 May, 2009. This figure is similar to Fig. 5, but in normal scale and not Kendrick mass scale. 